\title{
Exactly solvable non-Hermitian Jaynes-Cummings-type Hamiltonian admitting entirely real spectra from supersymmetry
}

\author{
Pijush K. Ghosh* \\ Theory Division, \\ Saha Institute of Nuclear Physics, \\ Kolkata 700 064, India.
}

\begin{abstract}
It is shown that for a given hermitian Hamiltonian possessing supersymmetry, there is always a non-hermitian Jaynes-Cummings-type Hamiltonian(JCTH) admitting entirely real spectra. The parent supersymmetric Hamiltonian and the corresponding non-hermitian JCTH are simultaneously diagonalizable. The exact eigenstates of these non-hermitian Hamiltonians are constructed algebraically for certain shape-invariant potentials, including a non-hermitian version of the standard Jaynes-Cummings(JC) model for which the parent supersymmetric Hamiltonian is the superoscillator. It is also shown that a non-hermitian version of the several physically motivated generalizations of the JC model admits entirely real spectra. The positive-definite metric operator in the Hilbert space is constructed explicitly along with the introduction of a new inner product structure, so that the eigenstates form a complete set of orthonormal vectors and the time-evolution is unitary.
\end{abstract}

*Electronic address: pijush@theory.saha.ernet.in 


\section{INTRODUCTION}

One of the standard axioms of quantum mechanics is to consider self-adjoint operators so that the corresponding eigenvalues are real and the time-evolution of the eigenstates is unitary. However, a new viewpoint emerging in the current literature is that although the condition of hermiticity is sufficient to have a unitary theory with real spectra, it is not necessary. The current development in this direction was boosted by the discovery of a class of non-hermitian Hamiltonians, invariant under the combined parity and time-reversal symmetry $(P T)$, that admit real spectra for unbroken PT symmetry 1, 2, 3, 4, 5, 6, 7, 8, 9, 10, 11, 12, 13, 14, 15, 16, 17, 18]. The spectra appear in complex-conjugate pairs, if the PT symmetry is broken spontaneously. This class of non-hermitian Hamiltonians with unbroken $P T$ symmetry is also shown to have a further symmetry $C$ similar to the

charge-conjugation [1]. The probabilistic interpretation of the quantum mechanics and the unitary time evolution of the eigenstates can be restored with the construction of a new inner-product using the $C P T$ symmetry. Field theoretical models with similar features have been studied in the literature[16].

A complementary approach in constructing physically meaningful theories with nonhermitian Hamiltonians admitting real spectra is to introduce the notion of pseudohermiticity [2]. An operator is said to be pseudo-hermitian, if it is related to its hermitian adjoint through a similarity transformation. The non-hermitian Hamiltonians admitting real spectra are shown to be pseudo-hermitian and are invariant under an anti-linear symmetry which reduces to the standard PT symmetry for some cases. A new inner product structure along with a positive-definite metric operator can be constructed in the Hilbert space using the property of pseudo-hermiticity. The probabilistic interpretation of the quantum mechanics and the unitary time evolution of the eigenstates can be restored with this new inner product.

The purpose of this paper is to construct a new class of non-hermitian Hamiltonians that admits entirely real spectra and allows an explicit construction of the positive-definite metric 
operator in the Hilbert space. In particular, we show that for a given hermitian Hamiltonian possessing supersymmetry, there is always a non-hermitian JCTH[19, 20] admitting entirely real spectra. The reality of the spectra is derived solely by using the superalgebra and without any particular representation of the supercharges. Moreover, the parent supersymmetric Hamiltonian and the corresponding non-hermitian JCTH can be diagonalized simultaneously. Thus, if the eigenstates of the parent supersymmetric Hamiltonian are known exactly, the eigenstates of the corresponding non-hermitian JCTH can be calculated easily. Among several known exactly/partly solved supersymmetric quantum mechanical Hamiltonians 21, 22, 23, 24], the eigenstates of non-hermitian JCTH corresponding to the one dimensional 'shape invariant' potentials are obtained exactly and algebraically. Only those 'shape invariant' potentials are considered for which the partner potentials are related to each other through a translation in the parameter. One of the most notable among these examples of non-hermitian JCTH is the standard JC Hamiltonian with non-hermitian interaction for which the superoscillator is the parent Hamiltonian. We show that the non-hermitian JC model considered in this paper admits a complete set of bi-orthonormal vectors [25]. Moreover, it can be shown that the non-hermitian JCTH is in fact pseudohermitian as well as quasi-hermitian[17, 18].

We also study a class of non-hermitian $2 \times 2$ dimensional matrix Hamiltonians that admits entirely real spectra. Special cases of this class of Hamiltonians include JC model with, intensity dependent coupling [26], Kerr nonlinearity [27], multi-photon interaction [28], q-oscillator [29] and dressed JC model 30]. We have also shown that a non-hermitian version of the Tavis-Cummings Model(TCM) [20], an N-molecule generalization of JC model, admits entirely real spectra. We explicitly construct the positive-definite metric operator in the Hilbert space and introduce the associated inner product structure for all these models. Consequently, the probabilistic interpretation of quantum mechanics and the unitary time evolution of the eigenstates can be retained.

The plan of this paper is the following. We first describe the superalgebra and introduce the non-hermitian Hamiltonian that admits entirely real spectra in the next section. We then 
find the expression for the energy eigenvalues of this non-hermitian Hamiltonian in terms of the energy eigenvalues of the parent supersymmetric Hamiltonian. In Sec. III, several examples of non-hermitian JCTH admitting entirely real spectra are studied. The example of a non-hermitian generalization of the standard JC Hamiltonian is studied in detail in Sec. III.A, followed by a general discussion on a non-hermitian $2 \times 2$ matrix Hamiltonian admitting entirely real spectra in Sec. III.B. We introduce and study a non-hermitian version of the TCM in Sec. III.C. A non-hermitian generalization of the supersymmetric Hamiltonians with 'shape-invariant' potentials for which the partner potentials are related to each other through a translation in the parameter is discussed in Sec. III.D. Finally, we conclude by summarizing our results in Sec. IV. In appendix A, the metric operator for non-hermitian $4 \times 4$ and $8 \times 8$ dimensional matrix Hamiltonian is constructed explicitly.

\section{JCTH FROM SUPERSYMMETRY: GENERAL FORMULATION}

The algebra governing the $\mathcal{N}=1$ supersymmetric quantum mechanics with the Hamiltonian $H$ is given by,

$$
\left\{Q, Q^{\dagger}\right\}=H, \quad Q^{2}=Q^{\dagger^{2}}=0, \quad[H, Q]=\left[H, Q^{\dagger}\right]=0
$$

where $Q$ is the supercharge and $Q^{\dagger}$ is its adjoint. The Hamiltonian $H$ is hermitian and semipositive definite by construction. All the eigenvalues $E_{n}$ of $H$ are thus real and semi-positive definite. We now construct a non-hermitian Hamiltonian $\mathcal{H}$ corresponding to $H$ as,

$$
\mathcal{H}=\left\{Q, Q^{\dagger}\right\}+c_{1} e^{i \theta} Q+c_{2} e^{-i \theta} Q^{\dagger}
$$

where $c_{1}, c_{2}$ and $\theta$ are real parameters. The Hamiltonian $\mathcal{H}$ is non-hermitian for $c_{1} \neq c_{2}$ and hermitian for $c_{1}=c_{2}$. The Hamiltonian $\mathcal{H}$ is identical to the JC model if $c_{1}=c_{2}$ and $Q$ is chosen to be that of one dimensional superoscillator. We thus refer to the whole class of $\mathcal{H}$ with arbitrary $Q$ and $c_{1,2}$ as JCTH. We will show that $\mathcal{H}$ admits entirely real spectra for

$$
\beta \equiv c_{1} c_{2}>0
$$


The spectra appears in complex conjugate pairs for $\beta<0$. No general conclusions could be drawn within our approach for the critical case $\beta=0$, i.e. either $c_{1}=0$ or $c_{2}=0$. We will be working in this paper only with $\beta$ obeying inequality (3).

Define an operator linear in the supercharges $Q$ and $Q^{\dagger}$,

$$
S(\theta) \equiv c_{1} e^{i \theta} Q+c_{2} e^{-i \theta} Q^{\dagger}
$$

The square of the operator $S$ is proportional to $H$,

$$
S^{2}=\beta H
$$

Although the operator $S$ is non-hermitian for $c_{1} \neq c_{2}$, its square $S^{2}$ is hermitian for any real $c_{1,2}$ and semi-positive definite for $\beta>0$. For hermitian $S$, i.e. $c_{1}=c_{2}$, the condition $\beta>0$ is satisfied automatically. The Hamiltonian $H$ and the operator $S$ can be diagonalized simultaneously, since they commute with each other. From Eq. (5), we find that the eigenvalues $E_{n}^{s}$ of $S$,

$$
E_{n}^{s}= \pm \sqrt{\beta E_{n}}
$$

are real, since $E_{n} \geq 0$ and $\beta$ is taken to be positive. For those cases for which $E_{n}$ has no upper bound, the operator $S$ is not bounded from below due to its negative eigenvalues. The eigenvalues of $\mathcal{H}=H+S$ are,

$$
\begin{aligned}
\mathcal{E}_{n}^{ \pm} & \equiv E_{n}+E_{n}^{s} \\
& =E_{n} \pm \sqrt{\beta E_{n}} .
\end{aligned}
$$

Although $\mathcal{H}$ is not hermitian, we have the remarkable result that its eigenvalues are real for $\beta>0$. Finally we remark that for $\beta<0$, the eigenvalues of $\mathcal{H}$ appear in complex conjugate pairs.

Following comments are in order.

(i) In supersymmetric quantum mechanics, the superalgebra with arbitrary $\mathcal{N}$ number of supercharges reads[31],

$$
\left\{Q_{a}, Q_{b}^{\dagger}\right\}=\delta_{a b} H, \quad\left\{Q_{a}, Q_{b}\right\}=\left\{Q_{a}^{\dagger}, Q_{b}^{\dagger}\right\}=0, \quad\left[H, Q_{a}\right]=0=\left[H, Q_{a}^{\dagger}\right], \quad a, b=1,2, \ldots \mathcal{N}
$$


Define the operator $S_{\mathcal{N}}$,

$$
S_{\mathcal{N}}=\sum_{a}\left(c_{1}^{a} e^{i \theta_{a}} Q_{a}+c_{2}^{a} e^{-i \theta_{a}} Q_{a}^{\dagger}\right)
$$

where $c_{1,2}^{a}$ and $\theta_{a}$ are real parameters for all $a$. We now find that $S_{\mathcal{N}}^{2}=\beta_{\mathcal{N}} H$ with $\beta_{\mathcal{N}}=$ $\sum_{a} c_{1}^{a} c_{2}^{a}$. Thus, the Hamiltonian $\mathcal{H}_{\mathcal{N}}=H+S_{\mathcal{N}}$ admits entirely real spectra for $\beta_{\mathcal{N}}>0$. The Hamiltonian $\mathcal{H}_{N}$ and $H$ can be diagonalized simultaneously, since $\left[H, S_{\mathcal{N}}\right]=0$.

(ii) There are many square-roots of the semi-positive definite operator $S^{2}$. First note that for any given non-hermitian square-root, its hermitian adjoint is also a square-root, since $S^{2}$ is hermitian. A few of these non-hermitian square-roots apart from $S$ are,

$$
\begin{aligned}
& S_{1}(\theta)=i \gamma_{5} S(\theta), \\
& S_{2}(\theta)=c_{1} e^{i \theta}\left(H+\epsilon^{2}\right) Q+c_{2} e^{-i \theta}\left(H+\epsilon^{2}\right)^{-1} Q^{\dagger}, \quad\{\epsilon \in \Re \mid \epsilon \neq 0\}, \\
& S_{3}=i \gamma_{5} S_{2},
\end{aligned}
$$

where $\gamma_{5}^{2}=1$ and it anticommutes with both $Q$ and $Q^{\dagger}$. A construction of $\gamma_{5}$ is given in appendix-A. The Hamiltonian $\mathcal{H}$ with $S$ replaced by any one of these $S_{i}$ 's, i.e. $\mathcal{H}_{i}=H+S_{i}$, would admit entirely real spectra for $i=1,2,3$. The Hamiltonian $\mathcal{H}$ and $\mathcal{H}_{i}$ are isospectral Hamiltonians. The eigenstates of $\mathcal{H}_{i}$ and $H$ can also be simultaneously diagonalized, since $\left[H, S_{i}\right]=0 \forall i$. However, a separate analysis is needed in each case for constructing the simultaneous eigenstates in the defining Hilbert space.

(iii) The relation between the JC model and supersymmetry was first noted in [32], where $c_{1}=c_{2}^{\dagger}$ was treated as an anticommuting variable instead of a $c$-number. This anticommuting variable also anticommutes with all the odd operators of the superalgebra and $c_{1}^{\dagger} c_{1}$ was chosen to be nilpotent in order to derive the spectrum. Thus, the approach taken in this paper in relating JC model to supersymmetry is different from that of Ref. 32]. Our approach is similar to that of Ref. [33] and the Hamiltonian $\mathcal{H}$ should be treated as a matrix Hamiltonian rather than an element of the superalgebra. However, the Hamiltonian $H$ is indeed a bosonic element of the superalgebra and the underlying superalgebra greatly simplifies the whole analysis. 


\section{EXAMPLES OF NON-HERMITIAN JCTH}

The superalgebra (11) for one dimensional quantum mechanical system can be realized in terms of the Pauli matrices $\sigma_{i}$ 's as,

$$
Q=\sigma_{+} a, \quad Q^{\dagger}=\sigma_{-} a^{\dagger}, \quad \sigma_{ \pm}=\frac{1}{2}\left(\sigma_{1} \pm i \sigma_{2}\right)
$$

where the operators $a$ and $a^{\dagger}$ are functions of the position and the momentum only. The Hamiltonian $\mathcal{H}$ now reads,

$$
\mathcal{H}=a^{\dagger} a+\frac{1}{2}\left[a, a^{\dagger}\right]\left(\sigma_{3}+1\right)+c_{1} e^{i \theta} \sigma_{+} a+c_{2} e^{-i \theta} \sigma_{-} a^{\dagger} .
$$

The Hamiltonian $\mathcal{H}$ and its adjoint $\mathcal{H}^{\dagger}$ are related to each other through the transformation $c_{1} \leftrightarrow c_{2}$. With the introduction of an operator $\eta$,

$$
\eta \equiv\left(\begin{array}{cc}
\delta_{1} & 0 \\
0 & \delta_{2}
\end{array}\right), \quad \frac{\delta_{1}}{\delta_{2}}=\frac{c_{2}}{c_{1}},
$$

it is easily verified that $\mathcal{H}$ is pseudo-hermitian, i. e. $\mathcal{H}^{\dagger}=\eta \mathcal{H} \eta^{-1}$. It may be noted that $\eta$ reduces to an identity matrix multiplied by a constant $\delta_{1}=\delta_{2}$ in the limit $c_{1}=c_{2}$ for which the hermiticity of $\mathcal{H}$ is restored. The matrix $\eta$ is not unique and among all possible such matrices, the unique positive-definite matrix $\eta_{+}$is obtained by taking $\delta_{1}=\gamma^{-1}$ and $\delta_{2}=\gamma$, where $\gamma$ is defined as $\gamma \equiv \sqrt{\frac{c_{1}}{c_{2}}}$. Note that the metric $\eta_{+}$is unique up to an overall multiplication factor that is positive-definite. The condition of pseudo-hermiticity implies that 2], if $|\psi\rangle$ is an eigenstate of $\mathcal{H}$ with the eigenvalue $\mathcal{E}$, then,

$$
|\phi\rangle=\eta|\psi\rangle
$$

is an eigenstate of $\mathcal{H}^{\dagger}$ with the same eigenvalue $\mathcal{E}$. Conversely, if $|\tilde{\phi}\rangle$ is an eigenstate of $\mathcal{H}^{\dagger}$

with the eigenvalue $\tilde{\mathcal{E}}$, then $|\tilde{\psi}\rangle=\eta^{-1}|\tilde{\phi}\rangle$ is an eigenstate of $\mathcal{H}$ with the eigenvalue $\tilde{\mathcal{E}}$. The relation (14) and the positive-definite matrix $\eta_{+}$will be used later to construct a new inner product in the Hilbert space. Further, $\mathcal{H}$ can be mapped to a hermitian Hamiltonian $h$,

$$
h=a^{\dagger} a+\frac{1}{2}\left[a, a^{\dagger}\right]\left(\sigma_{3}+1\right)+\beta\left(e^{i \theta} \sigma_{+} a+e^{-i \theta} \sigma_{-} a^{\dagger}\right)
$$

through the similarity transformation, $h=\rho \mathcal{H} \rho^{-1}$, where $\rho:=\sqrt{\eta}$ is the positive square-root of $\eta$. In the notion of Ref. [17, 18], $\mathcal{H}$ is also quasi-hermitian. 


\section{A. Non-hermitian resonant JC model}

The Hamiltonian $\mathcal{H}$ reduces to a non-hermitian generalization of the standard JC model, if $a, a^{\dagger}$ are chosen to represent the usual anhilation, creation operators of harmonic oscillators, i.e. $a=\frac{1}{\sqrt{2}}(p-i x), a^{\dagger}=\frac{1}{\sqrt{2}}(p+i x),\left[a, a^{\dagger}\right]=1$. The ground state has zero energy with the eigenstate, $\left|\psi_{0}\right\rangle=\gamma^{-\frac{1}{2}}\left[\begin{array}{c}0 \\ |0\rangle\end{array}\right]$, where $|n\rangle$ is the standard orthonormal basis of the harmonic oscillator with $a|n\rangle=\sqrt{n}|n-1\rangle, a^{\dagger}|n\rangle=\sqrt{n+1}|n+1\rangle,|n\rangle=\frac{\left(a^{\dagger}\right)^{n}}{\sqrt{n}}|0\rangle$ and $|0\rangle$ is the vacuum state annihilated by $a$. The excited states are,

$$
\mathcal{E}_{n+1}^{ \pm}=n+1 \pm \sqrt{\beta(n+1)}, \quad\left|\psi_{n+1}^{ \pm}\right\rangle=(2 \gamma)^{-\frac{1}{2}}\left[\begin{array}{c} 
\pm e^{i \theta} \gamma|n\rangle \\
|n+1\rangle
\end{array}\right], \quad n=0,1,2, \ldots
$$

The eigenvalues of the adjoint Hamiltonian $\mathcal{H}^{\dagger}$ are still given by $\mathcal{E}_{n+1}^{ \pm}$, while the corresponding eigenvectors $\left|\phi_{n+1}^{ \pm}\right\rangle$are obtained from $\left|\psi_{n+1}^{ \pm}\right\rangle$through the transformation $c_{1} \leftrightarrow c_{2}$ or using the Eq. (14) with $\eta_{+}$. In particular,

$$
\left|\phi_{n+1}^{ \pm}\right\rangle=(2 \gamma)^{-\frac{1}{2}}\left[\begin{array}{c} 
\pm e^{i \theta}|n\rangle \\
\gamma|n+1\rangle
\end{array}\right], \quad n=0,1,2, \ldots
$$

Note that the state $\left|\psi_{n}^{ \pm}\right\rangle$is not orthogonal to $\left|\psi_{m}^{\mp}\right\rangle$ with the usual definition of inner product, unless the condition of hermiticity, i.e., $c_{1}=c_{2}$ is imposed. However, the eigenstates $\left|\psi_{n}^{ \pm}\right\rangle$ and $\left|\phi_{m}^{ \pm}\right\rangle$together constitute a complete set of bi-orthonormal vectors for arbitrary $c_{1,2}$,

$$
\left\langle\psi_{n}^{I} \mid \phi_{m}^{J}\right\rangle=\delta_{m n} \delta_{I J}, \quad \sum_{n, I}\left|\psi_{n}^{I}\right\rangle\left\langle\phi_{n}^{I}\left|=\sum_{n, I}\right| \phi_{n}^{I}\right\rangle\left\langle\psi_{n}^{I}\right|=\mathbf{1}, \quad I, J=+,-
$$

A new inner product structure can be constructed as, $\langle\langle u \mid v\rangle\rangle_{\eta_{+}}=\left\langle u\left|\eta_{+}\right| v\right\rangle$. The norm of any arbitrary state vector $\langle u|=(\langle m|,\langle n|)$ is positive definite under this new inner product structure, since $\gamma$ is always positive definite for $\beta>0$. Furthermore, note that $\eta_{+}$reduces to an identity matrix in the limit $c_{1}=c_{2}$ for which the hermiticity of $\mathcal{H}$ is restored. Consequently, the new inner product structure is identical to the standard one for $c_{1}=c_{2}$. We now find a complete set of orthonormal vectors for $\mathcal{H}$,

$$
\left\langle\left\langle\psi_{n}^{ \pm} \mid \psi_{m}^{ \pm}\right\rangle\right\rangle_{\eta_{+}}=\delta_{n m}, \quad\left\langle\left\langle\psi_{n}^{ \pm} \mid \psi_{m}^{\mp}\right\rangle\right\rangle_{\eta_{+}}=0, \quad \sum_{m} \eta_{+}\left(\left|\psi_{m}^{-}\right\rangle\left\langle\psi_{m}^{-}|+| \psi_{m}^{+}\right\rangle\left\langle\psi_{m}^{+}\right|\right)=\mathbf{1}
$$

The Hamiltonian $\mathcal{H}$ is hermitian with respect to this new inner product. 
A comment is in order at this point. The operator $\gamma_{5}$ in Eq. (A2) is determined as $\gamma_{5}=-\sigma_{3}$ for $N=1$ and $\psi_{1}:=\sigma_{-}$. Note that $S_{1}\left(\theta+\frac{\pi}{2}\right)=S(\theta)$, implying that $\mathcal{H}_{1}$ and $\mathcal{H}$ are not independent of each other. Similarly, $\mathcal{H}_{2}$ and $\mathcal{H}_{3}$ are also not independent. However, it is expected that these Hamiltonians will be independent of each other for $N>1$.

\section{B. Non-hermitian non-resonant JC model \& other generalizations}

The example considered in the previous section corresponds to a non-hermitian generalization of the resonant JC model. A non-hermitian version of the non-resonant JC model also admits entirely real spectra. In particular,

$$
\mathcal{H}^{N R}=\mathcal{H}+\Delta \sigma_{3}
$$

is pseudo-hermitian under $\eta$ and admits entirely real spectra for $\beta>0$. For $\beta<0$, the eigenvalues are not entirely real and a further choice of $\theta=0$ reproduces the result obtained in Ref. [12]. The eigenvalues are,

$$
\mathcal{E}_{n+1}^{ \pm}=n+1 \pm\left[\Delta^{2}+\beta(n+1)\right]^{\frac{1}{2}}
$$

with the eigenstates,

$$
\left|\psi_{n+1}^{ \pm}\right\rangle=\left(\frac{\gamma}{\gamma^{2}+\left|\Gamma_{ \pm}^{n}\right|^{2}}\right)^{\frac{1}{2}}\left[\begin{array}{c}
\Gamma_{ \pm}^{n}|n\rangle \\
|n+1\rangle
\end{array}\right], \quad \Gamma_{ \pm}^{n} \equiv \frac{e^{i \theta}}{c_{2} \sqrt{n+1}}\left[\Delta \pm\left\{\Delta^{2}+\beta(n+1)\right\}^{\frac{1}{2}}\right]
$$

where $n$ is a non-negative integer. The ground-state energy is, $\mathcal{E}_{0}=-\Delta$ with the corresponding eigenstates, $\left|\psi_{0}\right\rangle=\gamma^{-\frac{1}{2}}\left[\begin{array}{c}0 \\ |0\rangle\end{array}\right]$. These eigenstates form a complete set of orthonormal vectors with respect to the new inner product structure $\langle\langle. \mid \cdot\rangle\rangle_{\eta_{+}}$.

There are many physically motivated generalizations of the standard JC model by including intensity-dependent coupling [26], Kerr nonlinearity [27], multi-photon interaction [28] and q-oscillator interaction [29]. In order to study a non-hermitian version of these generalized models, consider the following non-hermitian matrix Hamiltonian,

$$
\tilde{\mathcal{H}}=\left(\begin{array}{cc}
f_{1}\left(a, a^{\dagger}\right) & c_{1} e^{i \theta} g\left(a, a^{\dagger}\right) \\
c_{2} e^{-i \theta} g^{\dagger}\left(a, a^{\dagger}\right) & f_{2}\left(a, a^{\dagger}\right)
\end{array}\right), \quad f_{1}^{\dagger}=f_{1}, \quad f_{2}^{\dagger}=f_{2},
$$


where the hermitian adjoint is taken with respect to the standard inner product. The Hamiltonian $\tilde{\mathcal{H}}$ may or may not be supersymmetric for $c_{1}=c_{2}=0$. In general, $f_{1}, f_{2}$ and $g$ are arbitrary functions of the operators $a, a^{\dagger}$. Specific choices of $f_{1}, f_{2}$ and $g$ lead to integrable reductions of $\tilde{\mathcal{H}}$ that include a non-hermitian version of the generalized JC models [26, 27, 28, 29]. Note that the Hamiltonian $\tilde{\mathcal{H}}$ in its full generality is pseudo-hermitian, $\tilde{\mathcal{H}}^{\dagger}=\eta \tilde{\mathcal{H}} \eta^{-1}$, where $\eta$ is given by Eq. (13). Following Ref. [2], we conclude that $\tilde{\mathcal{H}}$ admits entirely real spectra for $\beta>0$, since the positive definite metric $\eta_{+}$exists for a positive definite $\beta$. Further, the new inner product structure $\langle\langle\cdot \mid \cdot\rangle\rangle_{\eta_{+}}$should be used for all relevant calculations. The Hamiltonian $\tilde{\mathcal{H}}$ is also quasi-hermitian, $\tilde{h}=\rho \tilde{\mathcal{H}} \rho^{-1}$, where $\tilde{h}$ is hermitian,

$$
\tilde{h}=\left(\begin{array}{cc}
f_{1}\left(a, a^{\dagger}\right) & \beta e^{i \theta} g\left(a, a^{\dagger}\right) \\
\beta e^{-i \theta} g^{\dagger}\left(a, a^{\dagger}\right) & f_{2}\left(a, a^{\dagger}\right)
\end{array}\right),
$$

and $\rho:=\sqrt{\eta}$. The eigen-spectra of $\tilde{\mathcal{H}}$ corresponding to the non-hermitian generalization of the models considered in Ref. [26, 27, 28, 29] could be obtained in a straightforward way.

The customary JC model is derived adopting the rotating-wave approximation[19]. There are additional terms in the Hamiltonian without this approximation and the corresponding Hamiltonian is known as "dressed" JC Hamiltonian in the literature[30, 32]. Note that a non-hermitian version of the "dressed" JC model is included in the Hamiltonian $\tilde{\mathcal{H}}$. In particular, if we choose,

$$
f_{1}=b_{1} a^{\dagger} a+b_{2}\left(a^{2}+a^{\dagger^{2}}\right)+b_{3}, \quad f_{2}=d_{1} a^{\dagger} a+d_{2}\left(a^{2}+a^{\dagger^{2}}\right)+d_{3}, \quad g=e_{1} a+e_{2} a^{\dagger},
$$

where $b_{i}, d_{i}, e_{i}$ are arbitrary real parameters, then $\tilde{\mathcal{H}}$ is a non-hermitian version of the "dressed" JC model that admits real spectra. All the discussions in the previous paragraph holds true for this Hamiltonian, except for the fact that finding the exact spectra is a non-trivial task. Finally, we would like to mention that for the special choices of the parameters,

$$
b_{1}=d_{1}=e_{1}^{2}+e_{2}^{2}, \quad b_{2}=d_{2}=e_{1} e_{2}, \quad b_{3}=e_{1}^{2}, \quad d_{3}=e_{2}^{2}
$$

the diagonal elements $f_{1}$ and $f_{2}$ correspond to the partner Hamiltonians of a supersymmetric Hamiltonian $\tilde{H}=\left\{\tilde{Q}, \tilde{Q}^{\dagger}\right\}$ with $\tilde{Q}=\sigma_{+} g, \tilde{Q}^{\dagger}=\sigma_{-} g^{\dagger}$. The Hamiltonian $\tilde{\mathcal{H}}$ can be expressed as, $\tilde{\mathcal{H}}=\tilde{H}+c_{1} e^{i \theta} \tilde{Q}+c_{2} e^{-i \theta} \tilde{Q}^{\dagger}$. 


\section{Non-hermitian Tavis-Cummings model}

The TCM deals with $N$ identical two-level molecules interacting through a dipole coupling with a single-mode quantized radiation field [20]. We consider a non-hermitian version of TCM,

$$
H_{T C}=a^{\dagger} a+R_{3}+\frac{1}{2}+c_{1} e^{i \theta} a R_{+}+c_{2} e^{-i \theta} a^{\dagger} R_{-}
$$

where the generators $R_{3}, R_{ \pm}$satisfy the $S U(2)$ algebra,

$$
\left[R_{3}, R_{ \pm}\right]= \pm R_{ \pm}, \quad\left[R_{+}, R_{-}\right]=2 R_{3}
$$

The hermitian TCM[20] is obtained in the limit $c_{1}=c_{2}$. Without loss of any generality, we have added an extra term equal to $\frac{1}{2}$ in the expression of $H_{T C}$ for the convenience of discussions in the later part of this section. The $S U(2)$ generators are realized in terms of the Pauli matrices and the $2 \times 2$ identity matrix $I$ as,

$$
\begin{aligned}
& R_{\mp}=\sum_{i=1}^{N} \Sigma_{\mp}^{i}, \quad R_{3}=\sum_{i=1}^{N} \Sigma_{3}^{i}, \\
& \Sigma_{a}^{i}=I \otimes \ldots \otimes I \otimes \sigma_{a} \otimes I \otimes \ldots \otimes I, \quad a=\mp, 3 \quad\left(\sigma_{a} \text { in } i-t h \text { position }\right) .
\end{aligned}
$$

We now define the metric operator $\eta_{N}$ as,

$$
\eta_{N}=\eta \otimes \eta \otimes \ldots \otimes \eta \quad(N \text { times })
$$

The Hamiltonian $H_{T C}$ is pseudo-hermitian under $\eta_{N}, H_{T C}^{\dagger}=\eta_{N} H_{T C} \eta_{N}^{-1}$. The positivedefinite metric operator $\eta_{N}^{+}$exists and it can be constructed through the replacement of $\eta$ by $\eta_{+}$in $\eta_{N}$. Thus, the non-hermitian TCM admits entirely real spectra with consistent quantum mechanical interpretation for $\beta>0$. The Hamiltonian $H_{T C}$ is quasi-hermitian under $\rho:=\sqrt{\eta_{N}}, h_{T C}=\rho H_{T C} \rho^{-1}$, where the hermitian $h_{T C}=a^{\dagger} a+R_{3}+\frac{1}{2}+\beta\left(e^{i \theta} a R_{+}+\right.$ $\left.e^{-i \theta} a^{\dagger} R_{-}\right)$

The individual two-level molecules in the TCM, with the $S U(2)$ generators given by Eq. (29), are independent of each other. However, the individual molecules are no longer independent of each other, if the representation of the $S U(2)$ generators given by Eq. (A4) 
in Appendix-A is used. The advantage of the representation of the $S U(2)$ generators given by Eq. (A4) is that the Hamiltonian $H_{T C}$ can now be written as,

$$
H_{T C}=\left\{Q, Q^{\dagger}\right\}+c_{1} e^{i \theta} Q+c_{2} e^{-i \theta} Q^{\dagger}, \quad Q=a^{\dagger} R_{-}, \quad Q^{\dagger}=a R_{+} .
$$

Defining the metric operator and its inverse as,

$$
\eta=\delta_{2} R_{+} R_{-}+\delta_{1} R_{-} R_{+}, \quad \eta^{-1}=\delta_{2}^{-1} R_{+} R_{-}+\delta_{1}^{-1} R_{-} R_{+},
$$

we find that $H_{T C}^{\dagger}=\eta H_{T C} \eta^{-1}$. The positive-definite metric exists and can be obtained by taking $\delta_{1}=\gamma^{-1}, \delta_{2}=\gamma$. Thus, the TCM with the representation of the $S U(2)$ generators given by Eq. (A4) also admits a real spectra with consistent quantum mechanical interpretation.

\section{Non-hermitian JCTH \& Shape-invariant potentials}

The operator $a, a^{\dagger}$ corresponding to more general supersymmetric Hamiltonian $H$ can be written as,

$$
a=p-i w(x, q), \quad a^{\dagger}=p+i w(x, q)
$$

where $q$ is a parameter and the superpotential $w$ is a real function of the coordinate $x$. The supersymmetric Hamiltonian $H$ and the corresponding non-hermitian JCTH $\mathcal{H}$ now reads,

$$
H=p^{2}+w^{2}+i \sigma_{3}[p, w], \quad \mathcal{H}=\Pi^{2}+W^{2}+i \sigma_{3}[\Pi, W]
$$

where the operators $\Pi$ and $W$ are defined as,

$$
\Pi \equiv p+\frac{c_{1} e^{i \theta}}{2} \sigma_{+}+\frac{c_{2} e^{-i \theta}}{2} \sigma_{-}, \quad W \equiv w(x, q)+\frac{c_{1}}{2} e^{i\left(\theta-\frac{\pi}{2}\right)} \sigma_{+}+\frac{c_{2}}{2} e^{-i\left(\theta-\frac{\pi}{2}\right)} \sigma_{-} .
$$

Both $\Pi$ and $W$ are non-hermitian for $c_{1} \neq c_{2}$ and $i \sigma_{3}[\Pi, W]=i \sigma_{3}[p, w]-\frac{c_{1} c_{2}}{2}$. The supersymmetric Hamiltonian $H$ is diagonal with the diagonal elements, $H_{ \pm} \equiv p^{2}+V_{ \pm}$, where the partner potentials $V_{ \pm}$are defined as, $V_{ \pm} \equiv w^{2} \pm \frac{\partial w}{\partial x}$.

We consider here only those cases for which $V_{ \pm}$are related to each other through a translation in the parameter $q$. Examples of such 'shape-invariant' potentials are abundant 
in the literature 21]. Following Ref. [22, 33], we further introduce the operators,

$$
T \equiv \exp \left(\xi \frac{\partial}{\partial q}\right), \quad T^{\dagger} \equiv \exp \left(-\xi \frac{\partial}{\partial q}\right), \quad B_{-} \equiv T^{\dagger}(q) a(q), \quad B_{+} \equiv a^{\dagger}(q) T(q)
$$

where $T$ is the translational operator for the parameter $q$. In particular, $T$ acting on an operator $O$ gives $T(q) O(q) T^{-1}(q)=O(q+\xi)$. Generalizing the algebraic method employed in 33. to the non-hermitian case, we find the eigenstates of $\mathcal{H}$,

$$
\mathcal{E}_{n+1}^{ \pm}=E_{n+1} \pm \sqrt{\beta E_{n+1}}, \quad\left|\psi_{n+1}^{ \pm}\right\rangle=(2 \gamma)^{-\frac{1}{2}}\left[\begin{array}{c}
e^{i \theta} \gamma T|n\rangle \\
\pm|n+1\rangle
\end{array}\right], \quad n=0,1,2, \ldots
$$

where $|n\rangle$ is an orthonormal basis for the generalized Heisenberg algebra satisfied by the operators $B_{ \pm}$with the role of number operator being played by $B_{+} B_{-} . E_{n}$ is eigenvalue of the operator $B_{+} B_{-}=a^{\dagger} a$. The ground state has zero energy with the eigenstate, $\left|\psi_{0}\right\rangle=$

$\gamma^{-\frac{1}{2}}\left[\begin{array}{c}0 \\ |0\rangle\end{array}\right]$. The eigenstates in Eq.(37) form a complete set of orthonormal vectors with the respect to the new inner product structure defined above.

\section{SUMMARY \& CONCLUSIONS}

We have shown that for a given hermitian Hamiltonian possessing supersymmetry, there always exists a non-hermitian JCTH admitting entirely real spectra. Moreover, if the parent supersymmetric Hamiltonian is exactly solvable, the corresponding JCTH can also be solved exactly. This is because these two Hamiltonians are simultaneously diagonalizable. These results are derived solely by using the superalgebra and without any particular representation for the supercharges. Thus, our prescription to construct non-hermitian Hamiltonian admitting entirely real spectra is very general and constitutes a new class itself.

We have also studied a class of non-hermitian $2 \times 2$ dimensional matrix Hamiltonians that admits entirely real spectra. Special cases of this class of Hamiltonians include JC model with, intensity dependent coupling, Kerr nonlinearity, multi-photon interaction, q-oscillator and dressed JC model. We have also shown that a non-hermitian version of the TCM, an $N$-molecule generalization of JC model, admits entirely real spectra. 
We have solved exactly the JCTH corresponding to one dimensional supersymmetric and shape invariant potentials. We have constructed a new inner product structure along with the positive-definite metric operator for this class of JCTH as well as for all other physically motivated generalizations of the JC model considered in this paper. Thus, for all these non-hermitian models, a complete set of orthonormal vectors exists and a consistent interpretation of the relevant physical observables are possible. Finally, the examples considered in this paper include a non-hermitian generalization of the standard JC model for which the parent supersymmetric Hamiltonian is the superoscillator. It would be nice if any observable

effect due to the non-hermiticity could be noticed for this Hamiltonian using micromaser 34] experiments.

\section{Acknowledgments}

This work is supported (DO No. SR/FTP/PS-06/2001) by SERC, DST, Govt. of India through the Fast Track Scheme for Young Scientists:2001-2002.

\section{APPENDIX A: EXPLICIT CONSTRUCTION OF THE METRIC OPERATOR $\eta$} FOR A MANY-PARTICLE SYSTEM

Consider a set of fermionic variables $\psi_{i}$ and $\psi_{i}^{\dagger}$ satisfying the Grassman algebra,

$$
\left\{\psi_{i}, \psi_{j}\right\}=\left\{\psi_{i}^{\dagger}, \psi_{j}^{\dagger}\right\}=0, \quad\left\{\psi_{i}, \psi_{j}^{\dagger}\right\}=\delta_{i j} .
$$

One can further define an operator $\gamma_{5}$,

$$
\gamma_{5}=(-1)^{N} \prod_{i=1}^{N}\left(2 \psi_{i}^{\dagger} \psi_{i}-1\right)
$$

which has the following property,

$$
\gamma_{5}^{2}=1, \quad\left\{\gamma_{5}, \psi_{i}\right\}=0=\left\{\gamma_{5}, \psi_{i}^{\dagger}\right\} \quad \forall i
$$


The matrix representation [35] of these anticommuting variables can be used to construct the generators of $S U(2)$. In particular,

$$
R_{-}=\frac{1}{\sqrt{N}} \sum_{i} \psi_{i}, \quad R_{+}=\frac{1}{\sqrt{N}} \sum_{i} \psi_{i}^{\dagger}, \quad R_{3}=\frac{1}{2 N} \sum_{i, j}\left[\psi_{i}^{\dagger}, \psi_{j}\right]
$$

Apart from satisfying the $S U(2)$ algebra (28), the generators (A4) also satisfy the following relations,

$$
R_{-}^{2}=0=R_{+}^{2}, \quad\left\{R_{-}, R_{+}\right\}=1, \quad R_{ \pm} R_{\mp}=\left( \pm R_{3}+\frac{1}{2}\right) .
$$

The generators in Eq. (A4) correspond to higher dimensional reducible representation of $S U(2)$.

The superalgebra (11) for higher dimensional or many-particle quantum mechanical system can be realized as,

$$
Q=\sum_{i=1}^{N} a_{i}^{\dagger} \psi_{i}, \quad Q^{\dagger}=\sum_{i=1}^{N} a_{i} \psi_{i}^{\dagger}
$$

The operators $a_{i}\left(a_{i}^{\dagger}\right)$ are expressed in terms of the momentum $p_{i}$ of the $i$ th particle and the superpotential $W(x)$,

$$
a_{i}=p_{i}-i W_{i}(x), \quad a_{i}^{\dagger}=p_{i}+i W_{i}(x), \quad W_{i}(x)=\frac{\partial W}{\partial x_{i}},
$$

where particle coordinates are denoted by $x_{i}$. Note that,

$$
\left[a_{i}, a_{j}\right]=0=\left[a_{i}^{\dagger}, a_{j}^{\dagger}\right], \quad\left[a_{i}, a_{j}^{\dagger}\right]=\frac{\partial^{2} W}{\partial x_{i} \partial x_{j}}
$$

The anti-commutation property of $\gamma_{5}$ in Eq. A3 implies that $\left\{Q, \gamma_{5}\right\}=0=\left\{Q^{\dagger}, \gamma_{5}\right\}$.

We now construct the metric operator $\eta$ for $N=2$ and $N=3$. For $N=2$, we choose,

$$
\psi_{1}=\sigma_{3} \otimes \sigma_{-}, \quad \psi_{2}=\sigma_{-} \otimes I
$$

The operators $\mathcal{H}, Q, S$ are $4 \times 4$ dimensional matrices with the elements being functions of $a_{1,2}$ and $a_{1,2}^{\dagger}$. It turns out that the metric operator $\eta$ is given by $\eta_{2}$ of Eq. (30). In a similar way, for $N=3$, we choose,

$$
\psi_{1}=\sigma_{3} \otimes \sigma_{-} \otimes I, \quad \psi_{2}=I \otimes \sigma_{3} \otimes \sigma_{-}, \quad \psi_{3}=\sigma_{-} \otimes I \otimes \sigma_{3}
$$


The operators $\mathcal{H}, Q, S$ are now $8 \times 8$ dimensional matrices with the elements being functions of $a_{1,2,3}$ and $a_{1,2,3}^{\dagger}$. We find the metric operator $\eta$ is given by $\eta_{3}$ of Eq. (30). Based on these results, we conjecture that for arbitrary $N$, the metric operator is given by $\eta_{N}$. The positive-definite metric operator $\eta_{N}^{+}$is obtained through the replacement of $\eta$ by $\eta_{+}$.

The Hamiltonian $\mathcal{H}$ admits entirely real spectra for $\beta>0$. Further, we have explicitly constructed the positive-definite metric operator for $N=2$ and $N=3$, implying that a consistent quantum mechanics can be constructed for these cases and for arbitrary $W(x)$. In fact, in two and three dimensions, one can now easily construct an exactly solvable non-hermitian $\mathcal{H}$ admitting entirely real spectra and with consistent quantum mechanical interpretation by simply choosing an exactly solvable $H$. Some of the simplest choices for $H$ are two and three dimensional superoscillators and all types of two and three particle super-Calogero models.

[1] C. M. Bender, quant-ph/0501052; C. M. Bender, D. C. Brody and H. F. Jones, Am. J. Phys. 71,1095 (2003) hep-th/0303005; C.M. Bender and S. Boettcher, Phys. Rev. Lett. 80, 5243(1998); J. Phys. A 31, L273(1998); C.M. Bender, S. Boettcher and P.N. Meisinger, J. Math. Phys. 40, 2210(1999).

[2] A. Mostafazadeh, J. Math Phys. 43, 205(2002); 43, 2814(2002); 43, 3944(2002); A. Mostafazadeh and A. Batal, J. Phys. A37, 11645(2004).

[3] P. Dorey, C. Dunning and R. Tateo, J. Phys. A 34, 5679(2001).

[4] M. Znojil, hep-th/0408081.

[5] B. Bagchi et. al., hep-th/0412211

[6] A. Mostafazadeh, Nucl. Phys. B640, 419 (2002).

[7] M. Znojil, F. Cannata, B. Bagchi and R. Roychoudhury, Phys.Lett. B483, 284 (2000).

[8] A. Andrianov, F. Cannata, J.-P. Dedonder and M.V. Ioffe, Int.J. Mod. Phys. A14, 2675 (1999).

[9] Z. Ahmed, Phys. Lett. A290, 19 (2001). 
[10] G. Japaridze, J. Phys. A35, 1709 (2002).

[11] S. Weigert, Czech. J. Phys. 54, 1139 (2004) quant-ph/0407132.

[12] B. P. Mandal, Mod. Phys. Lett. A20, 655(2005) hep-th/0412160.

[13] P. K. Ghosh, hep-th/0403083, To appear in Eur. Phys. J. C.

[14] P. K. Ghosh and K.S. Gupta, Phys. Lett. A323, 29(2004).

[15] B. Basu-Mallick, T. Bhattacharyya and B. P. Mandal, Mod. Phys. Lett. A20, 543 (2005)[ nlin.SI/0405068; B. Basu-Mallick, T. Bhattacharyya, A. Kundu and B. P. Mandal, Czech. J. Phys. 54, 5 (2004); B. Basu-Mallick and B.P. Mandal, Phys. Lett. A 284, 231 (2001); B. Basu-Mallick and A. Kundu, Phys. Rev. B62, 9927 (2000).

[16] C. M. Bender, S. F. Brandt, J.-H. Chen and Q. Wang, Phys. Rev. D71, 065010(2005) hep-th/0412316 ; C. M. Bender, D. C. Brody and H. F. Jones, Phys. Rev. D70, 025001(2004) hep-th/0402183; Erratum-ibid. D71 (2005); Phys. Rev. Lett. 93, 251601(2004) hep-th/0402011; C. M. Bender, S. Boettcher, H. F. Jones, P. Meisinger and M. Simsek, Phys. Lett. A291, 197(2001); C. M. Bender and K. A. Milton, Phys.Rev. D57, 3595(1998); C. M. Bender, F. Cooper, P. N. Meisinger and V. M. Savage, Phys. Lett. A259, 224(1999).

[17] F. G. Scholtz, H. B. Geyer and F. J. W. Hahne, Ann. Phys. 213, 74 (1992).

[18] R. Kretschmer and L. Szymanowski, Phys. Lett. A325, 112 (2004).

[19] E. T. Jaynes and F. W. Cummings, Proc. IEEE 51, 89 (1963).

[20] M. Tavis and F. W. Cummings, Phys. Rev. 170, 379 (1968).

[21] F. Cooper, A. Khare and U. Sukhatme, Supersymmetry in quantum mechanics, WorldScientific (Singapore), 2001.

[22] A. B. Blantekin, Phys. Rev. A57, 4188 (1998).

[23] P. K. Ghosh, Nucl. Phys. B681, 359(2004); T. Deguchi and P. K. Ghosh, J. Phys. Soc. Jap. 70, 3225 (2001); P. K. Ghosh, J. Phys. A34, 5583 (2001); P. K. Ghosh, Nucl. Phys. B595, 519 (2001); P. K. Ghosh, A. Khare and M. Sivakumar, Phys. Rev. A58, 821 (1998).

[24] E. D. Hoker and L. Vinet, Commun. Math. Phys. 97, 391 (1985).

[25] J. Wong, J. Math. Phys. 8, 2039 (1967); F. H. M. Faisal and J. Moloney, J. Phys. B14, 3603 
(1981).

[26] B. Buck and C. V. Sukumar, Phys. Lett. A81, 132 (1981).

[27] B. Deb and D. S. Ray, Phys. Rev. A48, 3191 (1993).

[28] C. C. Gerry, Phys. Rev. A37, 2683 (1988).

[29] M. Chaichian, D. Ellinas and P. Kulish, Phys. Rev. Lett. 65, 980 (1990).

[30] R. Gilmore and C. M. Bowdon, J. Math. Phys. 17, 1617 (1976).

[31] V. Akulov and M. Kudinov, Phys. Lett. B460, 365 (1999).

[32] C. Buzano, M. G. Rasetti and M. L. Rastello, Phys. Rev. Lett. 62, 137 (1989).

[33] A. N. F. Alexio, A. B. Balantekin and M. A. Cândido Ribeiro, J. Phys. A33, 3173 (2000); J. Phys. A34, 1109 (2001).

[34] D. Meschede, H. Walther and G. Müller, Phys. Rev. Lett. 54, 551(1985); G. Rempe and H. Walther, Phys. Rev. Lett. 58, 353 (1987); G. Rempe, F. Schmidt-Kaler and H. Walther, Phys. Rev. Lett. 64, 2783(1990).

[35] R. Coquereaux, Phys. Lett. B115, 389(1982). 